\title{
New Hands-on Fluid Mechanics Cartridges and Pedagogical Assessment
}

\section{Jacqueline K Burgher, Washington State University}

Jacqueline K. Burgher completed her undergraduate studies at Anderson University in Indiana in Chemistry and Mathematics-Economics. She then worked in water treatment for the city of Indianapolis, Ind. and earned an M.B.A. from Anderson University. Currently, she is an NSPIRE IGERT Trainee Ph.D. student in Chemical Engineering at Washington State University and working under Bernie Van Wie on hands-on learning solutions for students.

\section{Mr. David Finkel, Washington State University \\ Mr. Bernard J. Van Wie, Washington State University}

Prof. Bernard J. Van Wie did his B.S., M.S. and Ph.D., and postdoctoral work at the University of Oklahoma where he also taught as a visiting lecturer. He has been on the Washington State University faculty for 30 years and for the past sixteen years he has focused strongly on innovative pedagogy and done technical research in biotechnology. His recent Fulbright exchange to Nigeria set the stage for him to receive the Marian Smith Award given annually to the most innovative teacher at Washington State University.

\section{Dr. Olusola Adesope, Washington State University-Pullman}

Dr. Olusola O. Adesope is an assistant professor of Educational Psychology at Washington State University at Pullman. His research is at the intersection of educational psychology, learning sciences, and instructional design and technology. His recent research focuses on the cognitive and pedagogical underpinnings of learning with computer-based multimedia resources; knowledge representation through interactive concept maps; meta-analysis of empirical research, and investigation of instructional principles and assessments for engineering designs. Dr. Adesope holds a Ph.D. in Educational Psychology and M.Sc. in Educational Technology from Simon Fraser University, Canada.

\section{Dr. Shane A. Brown P.E., Washington State University}

Dr. Shane Brown conducts research on cognition and conceptual change in engineering. He received his bachelor's and Ph.D. degrees from Oregon State University, both in civil engineering. His Ph.D. degree includes a minor in science and mathematics education. His master's degree is in environmental engineering from the University of California, Davis. Dr. Brown is a licensed professional civil engineer and has six years of experience designing water and waste water treatment facilities in central California. He was the recipient of the NSF CAREER award in 2011. Dr. Brown's research interests are in conceptual change, epistemology, and social or situated cognition. Specifically, his research focuses on theoretical approaches to understanding why some engineering concepts are harder to learn than others, including the role of language and context in the learning process.

\section{Mr. Justin William Atkinson}




\section{New Hands-on Fluid Mechanics Cartridges and Pedagogical Assessment}

Abstract: In engineering and the sciences, effectively communicating concepts and fundamental ideas to students is difficult with traditional teaching methodologies. Demonstration-mode teaching pedagogy has been used at our university in the past to address this problem within a fluid mechanics and heat transfer class. To assess learning gains and persistent misconceptions, we conducted interviews with eight students from a range of GPAs who had previously taken the course. The interview questions were developed using Bloom's taxonomy, and the interview results were coded using a qualitative software program. Results from the analysis indicate conceptual difficulties primarily with K-value loss coefficients, noncircular channels, and the mechanical energy balance and secondarily with flow regimes and energy transformations in a Venturi meter. There was least concern about conceptual understanding related to pressure losses in fittings. Results subsequently informed the design of learning activities, assessment materials, and the reformatting of two miniature hands-on cartridges used in desktop learning modules (DLMs). Cartridges include clear viewing windows and snap into a base unit used to modulate flow rate and display pressure drops. One cartridge is designed for measuring pressure drop through a straight pipe, shallow bend, and $90^{\circ}$ miter bend, a second with another with a $180^{\circ}$ bend; and a third with a venturi meter designed to assist understanding of energy transitions. Using these materials, a within design study included about 40 students: 20 who served as a control group for a first set of concepts taught by lecture, while another 20 who received a mini orientation-lecture, participated in a hands-on active learning session with DLMs, discussed an in-class worksheet in groups to process material content, and then completed the worksheet as an individual homework assignment. For a second set of concepts, the two groups were switched. Pre- and post-conceptassessments are being used to measure differences between control and experimental groups. The experimental group is expected to gain a better understanding of the fundamental concepts, and preliminary results will be reported in the ASEE presentation.

\section{Introduction}

Traditional lecture methods continue to produce engineering students with deficiencies in conceptual understanding ${ }^{3}$. Addressing these misconceptions is imperative to amend the gaps in engineering education, and other teaching methodologies have been shown to better equip students for long-term conceptual understanding ${ }^{1,2}$. Identification of these misconceptions can help in the design of hands-on and cooperative learning strategies to increase student learning. In addition, instruction that involves hands-on learning activities enables the students to develop long-term problem solving techniques and drives their interest in the subject matter. ${ }^{3}$

Fluid mechanics remains an important field of study in several disciplines, including chemical engineering. Applications include proper understanding and analysis of flow regimes and profiles, velocity and pressure changes related to energy transitions and losses through generic fittings, and specialized contractions like Venturi and orifice meters. Student learning of these concepts is imperative for comprehensive understanding of fluid mechanics. Use of traditional lecture consistently demonstrates its inability to address student misconceptions, which is why other teaching pedagogies and hands-on learning methods need to be implementated ${ }^{2,3}$. In a companion study, our colleagues in civil engineering have achieved success in reducing 
conceptual difficulties by incorporating hands-on learning modules and designing interactive learning experiences targeting common misconceptions. ${ }^{7,8}$ Within chemical engineering, we have made many advances to include hands-on experiments with electrophoresis, ${ }^{6}$ desktop experimental modules, ${ }^{9}$ and a 15 year effort at Washington State University (WSU) to develop and implement Desktop Learning Modules (DLMs) ${ }^{5}$. Until recently, however, we have not seen a dedicated effort to design DLM learning activities specifically around persistent misconceptions and demonstrated that these activities contribute to student conceptual gains. In this paper, we present the first definitive steps to rigorously interviewing students who already have taken fluid mechanics and heat transfer, identify and categorize remaining conceptual difficulties, and reformat DLM cartridges and associated activities to rectify the issues and assess impacts.

\section{Methods}

This study first identifies the persisting conceptual difficulties in undergraduate student understanding after having completed a fluid mechanics course; this is achieved through interviews of the students. Upon identification and classification utilizing Bloom's taxonomy and qualitative software analysis, these conceptual difficulties are used to develop a worksheet to accompany a bends and pipes and a Venturi meter hands-on DLM. A within-design study is then applied to a fluid mechanics class, with one group receiving treatment using the hands-on learning bends and pipes module and the other section receiving treatment with the Venturi meter. In both cases, the untreated group receives traditional lecture as opposed to a hands-on experience. The timeline of this study can be viewed in Table 1.

Table 1: Timeline outlining individual tasks performed for this study.

\begin{tabular}{|c|l|}
\hline \multicolumn{2}{|c|}{ Timeline and Methods } \\
\hline Task & \multicolumn{1}{|c|}{ Objective } \\
\hline $\begin{array}{c}\text { Structured } \\
\text { Interviews }\end{array}$ & $\begin{array}{l}\text { Gather conceptual knowledge of persisting conceptual difficulties in students } \\
\text { who have completed a fluid mechanics course. }\end{array}$ \\
\hline $\begin{array}{c}\text { Interview } \\
\text { Analysis }\end{array}$ & $\begin{array}{l}\text { Code, classify, and rank concepts and conceptual difficulties for the fluid } \\
\text { mechanics course. }\end{array}$ \\
\hline $\begin{array}{c}\text { Worksheet } \\
\text { Design }\end{array}$ & $\begin{array}{l}\text { Create learning tools to address and amend the persisting conceptual } \\
\text { difficulties found in students' understanding based on identified conceptual } \\
\text { difficulties. }\end{array}$ \\
\hline $\begin{array}{l}\text { Within-Design } \\
\text { Study }\end{array}$ & $\begin{array}{l}\text { Analyze pre- and post-test assessments for 41 students (half given the } \\
\text { treatment, half a lecture) for bends and pipes and Venturi meter cartridges. }\end{array}$ \\
\hline
\end{tabular}

\section{Materials}

\section{Desktop Learning Modules (DLMs)}

The DLM is a hands-on base unit with accompanying cartridges that can be snapped into and out of the unit. An electronic screen present on the base unit enables students to read pressure drops and temperatures from the cartridge snapped into the unit. Flow rates are adjusted and read with a rotometer for older DLMs, while they are measured with an in-line flow meter and displayed on the screen for newer DLMs. 
The bends and pipes cartridge is used for measuring pressure drop with fluid flow rates through a straight pipe, shallow bend, and $90^{\circ}$ miter bend for one version of the cartridge and in a straight pipe and $180^{\circ}$ bend for another version. Velocity and pressure drop for each respective bend is then read from the screen on the base unit; calculations can be done from these values and be compared with theoretical results. The design of the bends and pipes cartridge enables students to compare the pressure loss between various types of fittings. Equal lengths of pipe for the straight section, shallow bend, and $90^{\circ}$ miter or $180^{\circ}$ bend offer students the ability to numerically associate pressure drops with the respective bend by subtracting the pressure drop for the straight section. This association may have the ability to clarify and reinforce conceptual ideas that accompany the physical phenomena. The photo in Figure 1 shows the $180^{\circ}$ bend cartridge.

The Venturi meter is used to demonstrate the relationship between pressure and velocity through a contraction and expansion. The DLM-cartridge system displays the flow rate and pressure drop across the meter. The pressure tap locations are strategically placed, allowing students to compare pressure drop through the contraction and pressure recovery after a gentle $15^{\circ}$ expansion. Concepts of continuity and the relationship between $\Delta \mathrm{P} / \rho$, or flow work energy term, and kinetic energy changes in with varying cross sectional area may be communicated with this cartridge. The Venturi meter cartridge, shown in Figure 2, is designed in a similar fashion as the energy loss in bends and pipes cartridge with leak proof snap-in connectors and a hypodermic syringe Luer-Lok ${ }^{\mathrm{TM}}$ fitting and needle port that allows dye injection into the centerline of flow for conducting the Reynolds experiment.

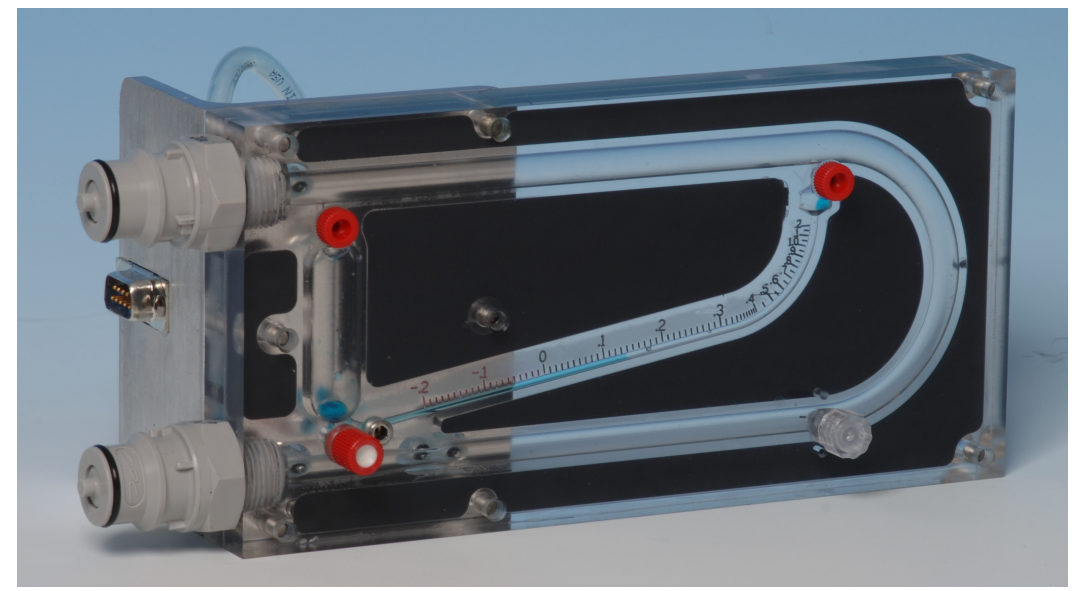

Figure 1: Modified cartridge useful for focusing on energy loss through a $180^{\circ}$ bend and straight section. 


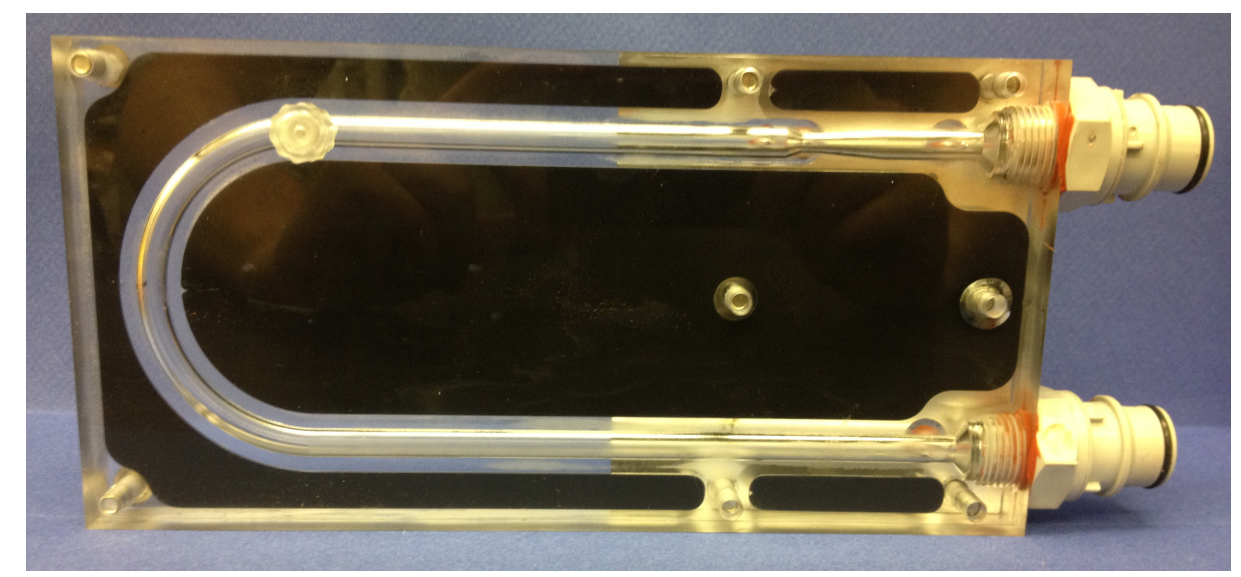

Figure 2: Venturi meter cartridge with pressure readings over entire cartridge and at the beginning, center of the throat, and end of the meter.

Interview Materials and Worksheets

Investigation into student conceptual understanding enables and enhances the development of appropriate worksheets targeted to amend incorrect thought processes of students. First, it was necessary to determine persisting conceptual difficulties of students who had completed the fluid mechanics class, so an interview protocol was developed. The structure was modeled after a study conducted on open channel flow, which incorporated qualitative interview results into the development of assessment materials to accompany hands-on learning cartridges. ${ }^{5}$ The process began with a search on previously identified misconceptions in fluid mechanics. Initial findings indicate categories surrounding the Bernoulli equation, viscous momentum transfer, and mass conservation in fluid systems are difficult but important fluid mechanics principles for students. ${ }^{10}$

These findings were then summarized and reviewed by two professors with experience teaching the course. Their input and feedback identified seven major areas where students struggle which could be addressed with the hands-on learning modules. The concepts identified include flow regimes, the mechanical energy balance, the Venturi meter, continuity and pressure drop through a piping system, pressure loss through fittings, K-value loss coefficients, and analysis of noncircular channels. These topics created the outline for the interview protocol; follow-up questions aimed to capture incorrect thinking and reinforced the aforementioned concepts. Upon completion, a draft of the protocol underwent six iterations to ensure correct phrasing and clear figures that accompanied questions. A key was developed alongside the protocol, which helped the interviewer maintain a clear understanding of the concepts associated with each question; this enabled the pursuit of follow-up prompts if a student was struggling with an answer. Student responses would help determine whether or not the students actually knew the content. Examples included questions about missing terms in the mechanical energy balance or contradictory statements about the Venturi meter. This also allowed us to draw conclusions about a student's conceptual understanding with respect to disciplinary vocabulary, and if this contributes to conceptual difficulty in a student's thinking.

The representative sample of students selected from the chemical engineering fluid mechanics and heat transfer course had previous exposure to the desktop learning modules (DLMs) and accompanying cartridges in the form of a demonstration only with the use of a document camera. Throughout the course, visuals accompanied relevant lecture materials six times. These 
demonstrations included a Reynolds experiment with dye injection, orifice plate, venturi meter, fluidized bed, shell and tube heat exchanger, and double pipe heat exchanger. Data for each experiment was gathered during demonstration, and students were assigned individual worksheets as homework to complete for each learning experience.

Eight interview candidates from a large research university in the northwest were selected for this study. All had previously taken and passed the fluid mechanics and heat transfer course. The candidates were selected based on GPA; two with a GPA 3.5 or above, three with a GPA between 3.0-3.5, and three with GPAs below 3.0. This study used a structured interview and a few optional questions depending on the responses of the interviewee and discretion of the facilitator. These questions were pre-determined before the interview.

It is important to note the time elapsed from the end of the course to the interviews. Approximately six months passed between the final lecture and the interviews; these interviews were used to assess the concepts retained after that period. Upon completion of the interviews, recordings were transcribed and analyzed using the qualitative data analysis software Atlas ti (ATLAS.ti Scientific Software Development GmBH, Berlin, Germany). Codes were established to determine correct or incorrect answers to questions. If incorrect, the response was tagged to identify if the student was dispelling erroneous information, the information was partially correct but incomplete with the student omitting an essential fundamental idea, or the information included correct information but demonstrated incomplete understanding by adding incorrect information.

The complete interview protocol is in Table 2; the questions are listed with the associated fluid mechanics concepts under each major category. Because these interviews are structured, the questions were asked verbatim to students with each receiving a packet of figures to complete during the course of the interview. These diagrams also appear in Table 2 and were analyzed alongside the responses with the qualitative analysis software.

Table 2: Interview protocol with the concepts listed and corresponding questions related to each concept asked of each interviewee. The letter in brackets before each question identifies which category of Bloom's hierarchy the question addresses: $K$-knowledge, $C$-comprehension, $A-$ application.

\begin{tabular}{|c|c|}
\hline \multicolumn{2}{|r|}{ Interview Protocol } \\
\hline Concept & Questions Asked \\
\hline $\begin{array}{l}\text { Flow Regimes } \\
\text { Concepts: } \\
\text { - Laminar and } \\
\text { Turbulent Flow } \\
\text { - Convective and } \\
\text { Viscous Forces } \\
\text { - Transition in Flow } \\
\text { Regime } \\
\text { - Flow Regime } \\
\text { Impact on Frictional }\end{array}$ & $\begin{array}{l}\text { A) [K] What are the main regimes of flow? } \\
\mathrm{B})[\mathrm{C}] \text { Can you provide a detailed answer on how they are different? } \\
\text { C) [C] Draw a representative section of pipe containing each type of } \\
\text { flow and explain the differences. }\end{array}$ \\
\hline
\end{tabular}




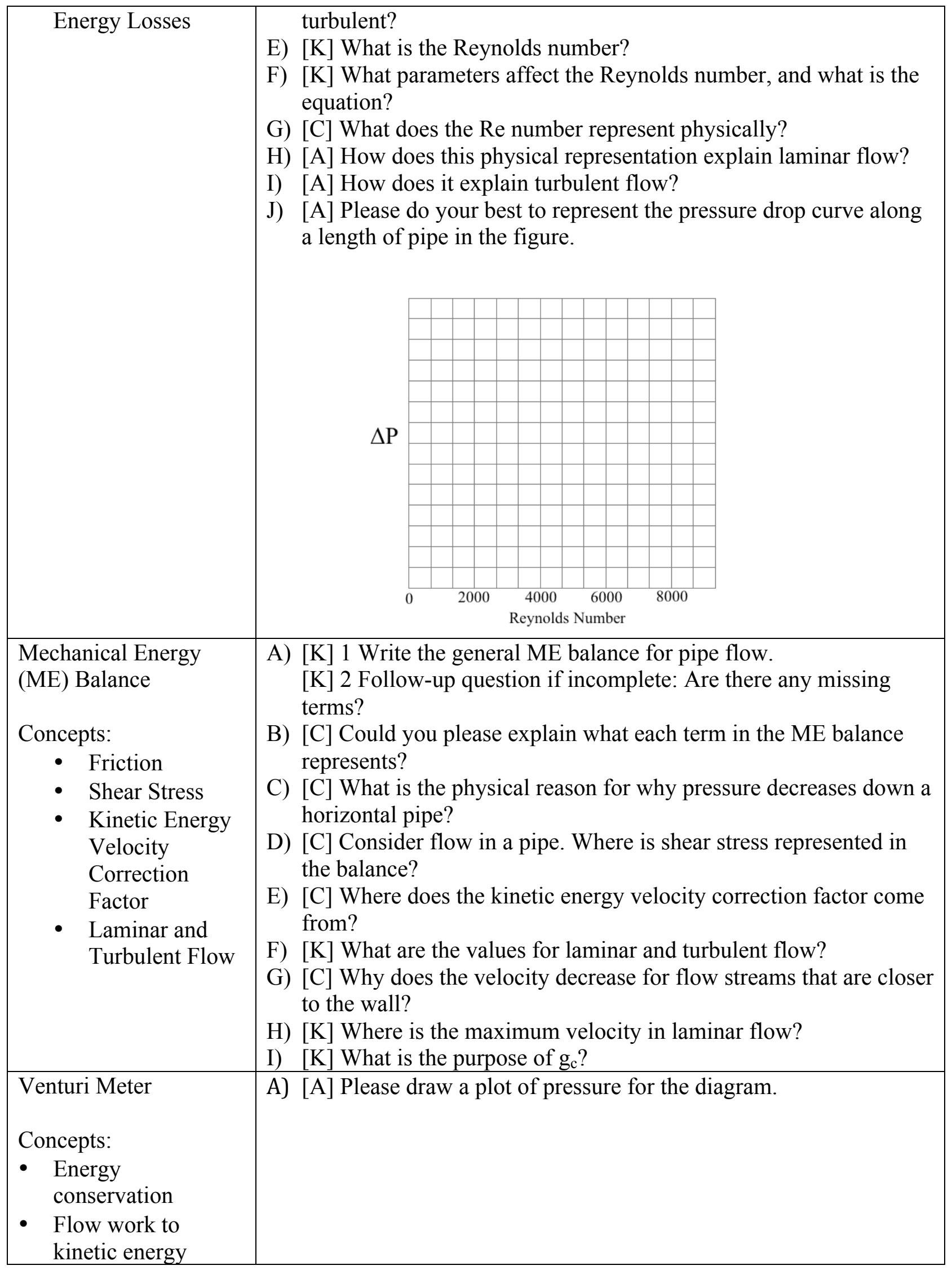


transformations

- Conservation of mass/ continuity

- Isentropic contraction
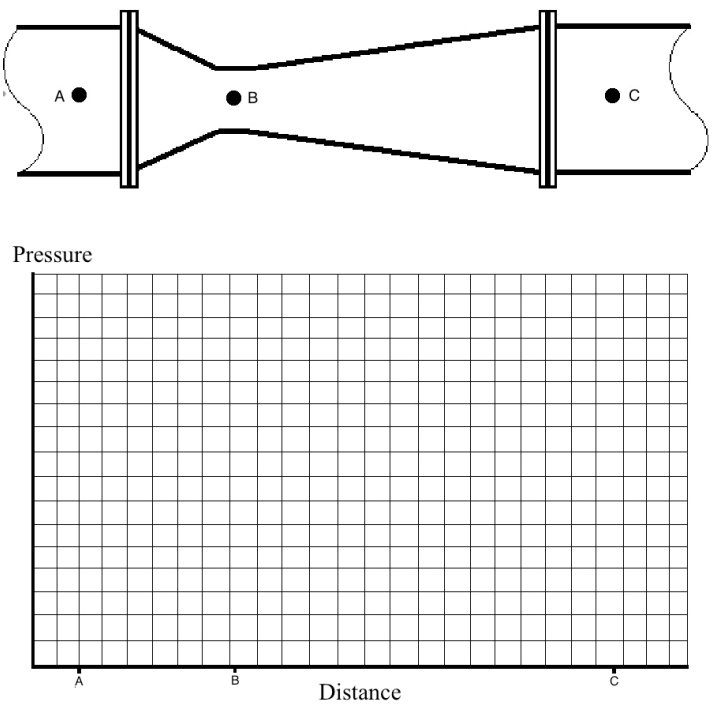

B) $[\mathrm{A}]$ Please draw a plot of velocity for the diagram.
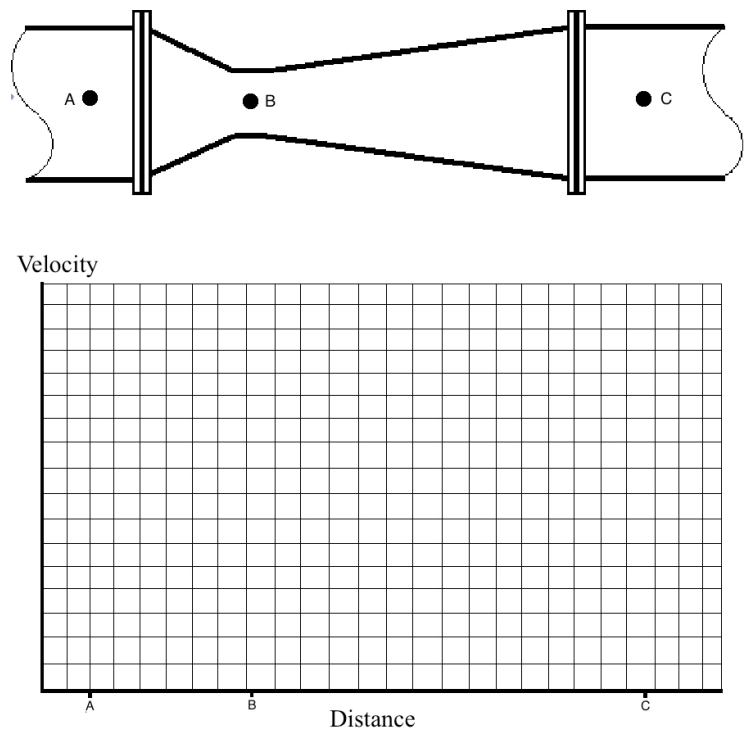

Apply the ME balance to the following pipe segments in the diagram and justify removal of any terms.

C) $[\mathrm{A}] \mathrm{A} \rightarrow \mathrm{B}$

D) [A] What energy quantities change and in which direction, i.e. increase (positive) or decrease (negative)?

E) [C] What would be the difference in total energy between points $\mathrm{A} \rightarrow \mathrm{B}$ assuming no frictional losses?

F) [A] Why does velocity change through the throat?

G) $[A]$ For pipe segment $A \rightarrow C$ ?

$\mathrm{H})$ [A] Which energy quantities change and in what direction?

I) [C] There are often energy losses in contractions and expansions. Why are these minimal in the Venturi meter? 


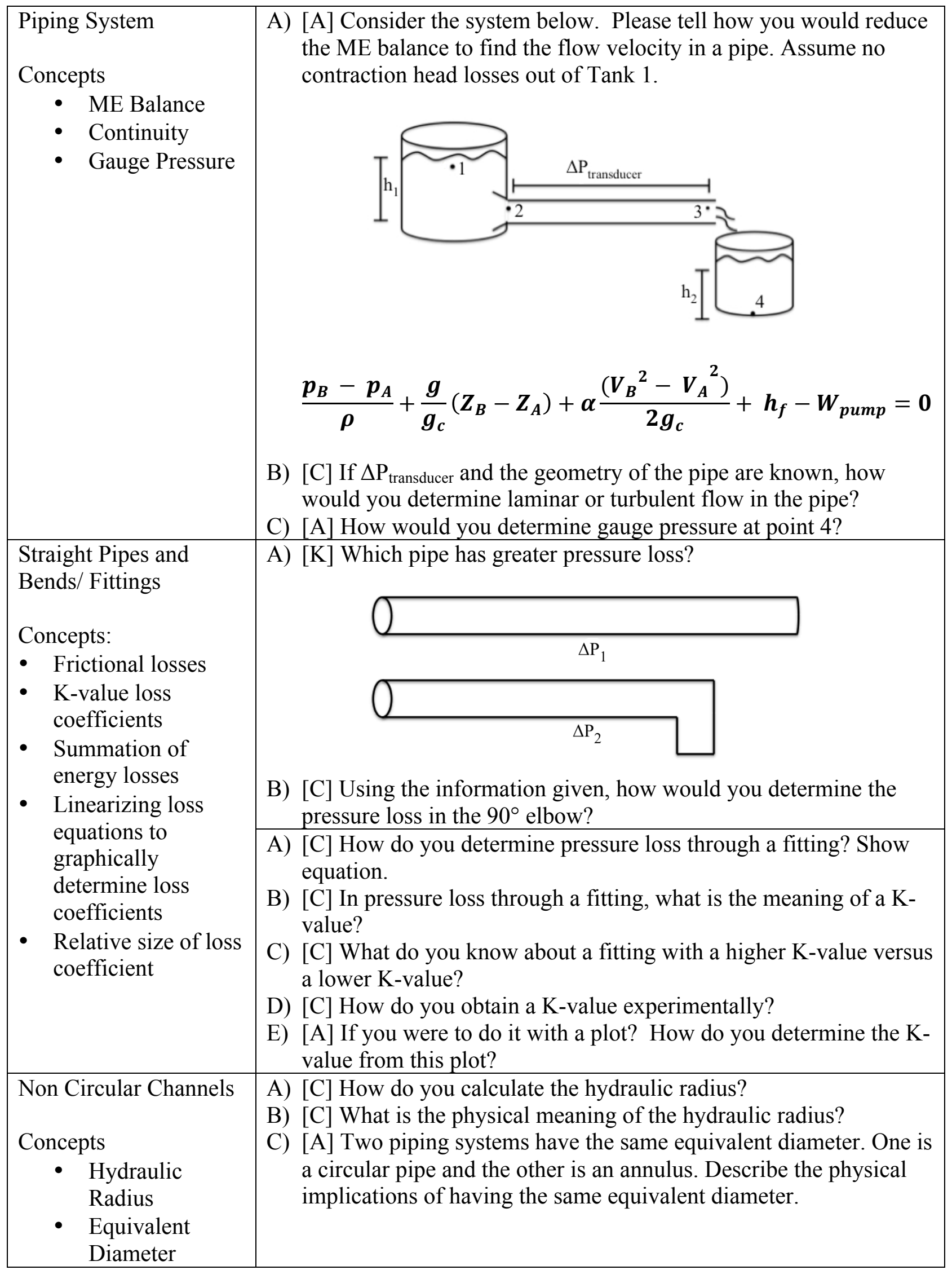




\section{Analysis \& Results}

Analysis of the interview responses was completed with reference to The Taxonomy of Educational Objectives developed by Bloom and collegues ${ }^{4}$. This consists of six levels of learning: knowledge, comprehension, application, analysis, synthesis, and evaluation. Each question was characterized according to this taxonomy, with all the questions in the protocol categorized by the first three tiers: knowledge, comprehension and application. These were assigned the corresponding letters $\mathrm{K}, \mathrm{C}$, and $\mathrm{A}$, respectively, which allows classification of the type of learning associated with each question. These designations were placed in parentheses after each question in the interview list. Knowledge questions deal with basic facts that require little understanding and rote memorization. Comprehension questions ask why and require students to describe physical phenomena by paraphrasing ideas. Analysis is the furthest up the hierarchy questions; it requires making connections between ideas and correctly applying them to solve complex problems. ${ }^{3,4}$

The results from the taxonomy classification of questions can be viewed in Figures 1, 2, and 3, with the largest percentage of correct responses occurring for knowledge questions and the most percent incorrect from the application.

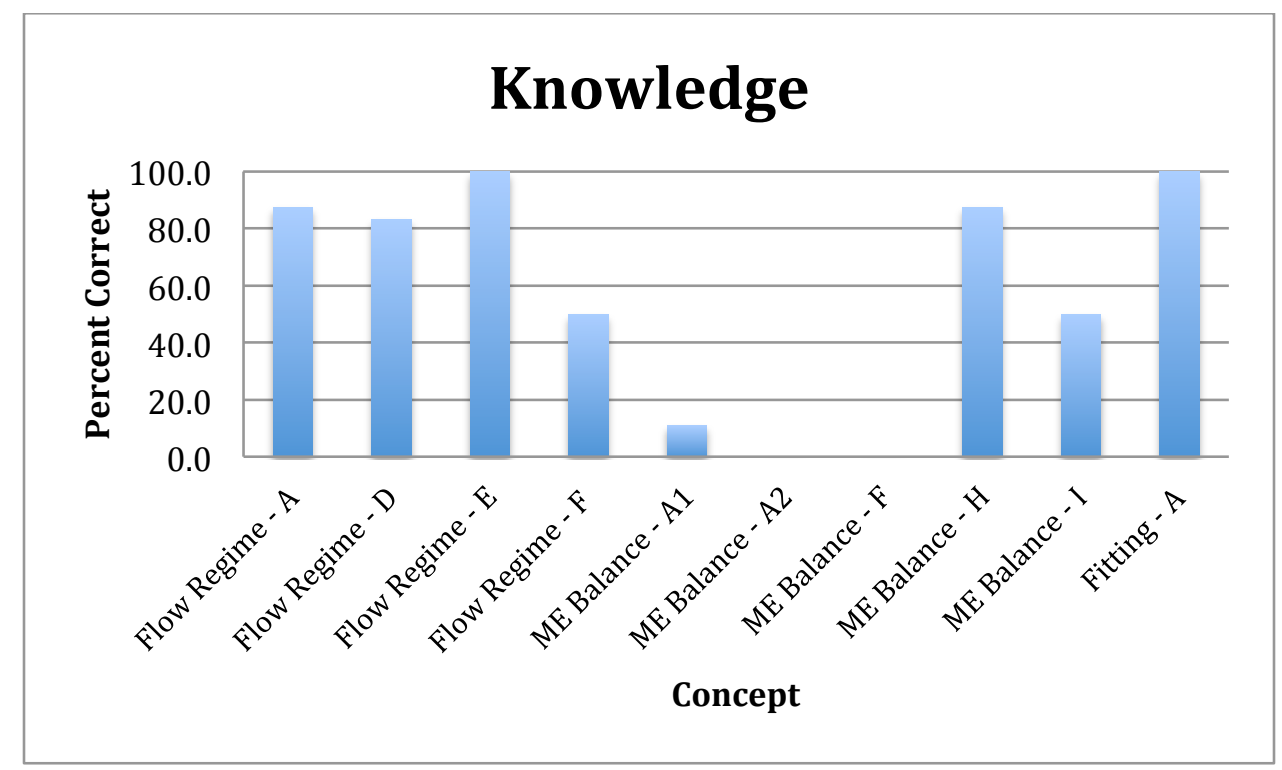

Figure 2: Percent correct answers of knowledge questions asked in the interviews. Letters correspond to the interview questions under each category, identified in Table 2.

Knowledge questions are the first tier on Bloom's taxonomy and test the most basic level of student conceptual understanding. Based on the results in Figure 1, we conclude students retained facts about flow regimes and fittings quite well with over $80 \%$ correct on most questions except Question F which showed 50\% correct. However, retention of knowledge about the mechanical energy balance was much more challenging with the percentage correct in the 0 $10 \%$ range for Questions A and F and 50\% for Question $\mathrm{H}$. In Question A, students were asked to write the energy balance, with only one successfully including all terms. When followed up 
with a subsequent question regarding missing terms, none of the students could identify the terms they missed.

Comprehension questions consist primarily of understanding physical phenomena and describing these with paraphrased ideas. Most of the questions on the protocol fell into this category, and performance was much lower than anticipated. Only two of the seventeen questions were answered over $80 \%$ correct; the remaining 15 questions all scored below a $67 \%$. The strongest retention in students occurred with facts about total energy loss through a Venturi meter and how to determine pressure through a $90^{\circ}$-miter bend given two pipes of the same length: one straight pipe and one pipe with a $90^{\circ}$-miter bend.

Interview results indicate students had the most difficulty with K-value loss coefficients in the comprehension category. Given four questions, average total correct was $27 \%$, with the most difficulty coming with the first question of how to determine pressure loss through a fitting. These results indicate students have not retained how K-values relate to the piping systems, the physical phenomena relevant to the analysis.

Students also performed poorly on the ME balance questions. Given four questions, students only answered $30 \%$ correct with every student unable to identify the terms that account for shear stress in the system. Similarly, questions about noncircular channels scored an average of 35\% correct, indicating students did not properly retain information on how to analyze these systems. Questions regarding flow regimes gave stronger results; one question had $60 \%$ correct, a second had $45 \%$, and the third had $38 \%$ correct.

Results from comprehension in Bloom's taxonomy indicate decreasing understanding as the type of question changes and gets more difficult; interviews are prime opportunities for students to articulate their understanding, but few were able to accurately answer the questions.

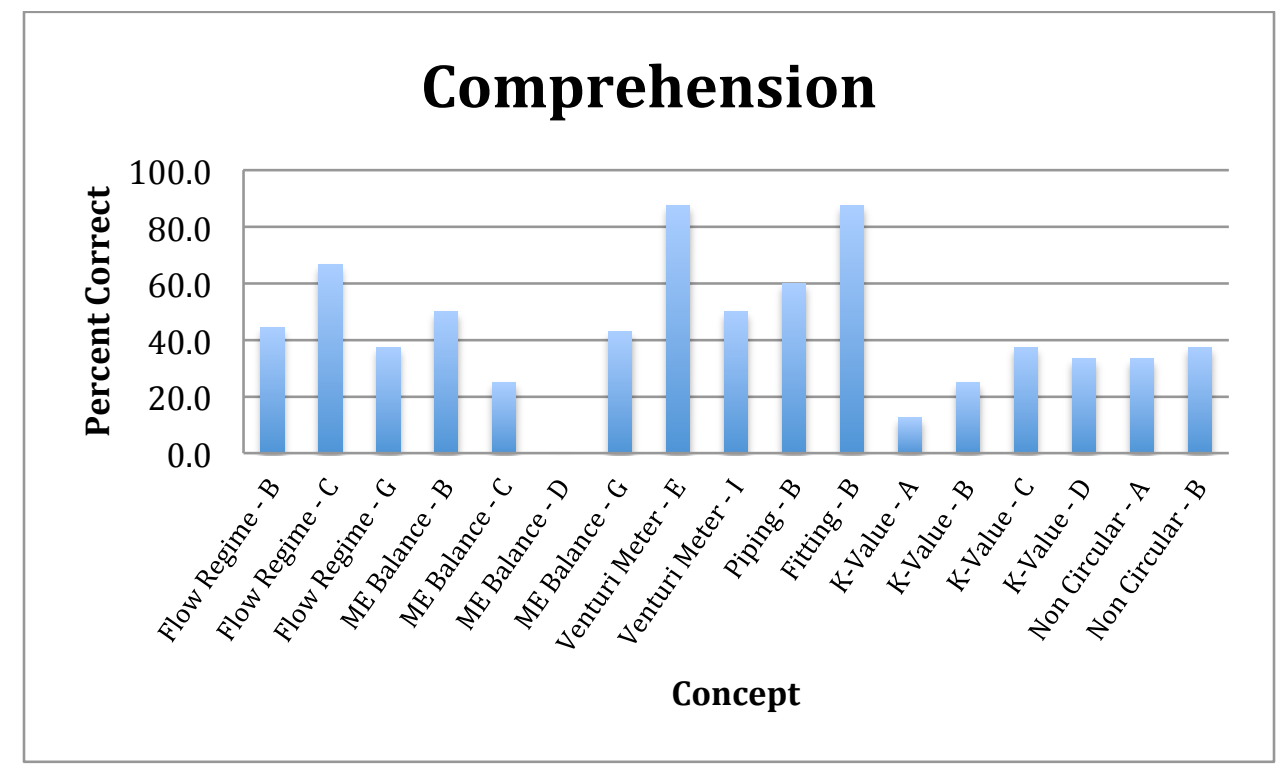

Figure 3: Percent correct answers of comprehension questions asked in the interviews. Letters correspond to the interview questions under each category, identified in Table 2. 
The application questions proved the most difficult for students. Collectively, the group did not achieve over $60 \%$ correct for any of the conceptual questions. Breaking these down into categories, the two lowest scoring were flow regimes with $12.5 \%$ correct on every question and noncircular channels with an average of $6 \%$ correct. These were followed closely by the K-value energy loss coefficient Question E that had 20\% correct. Answers for the Venturi meter were better with the average 29\% correct and the piping system performing the best with Question B receiving $50 \%$ correct.

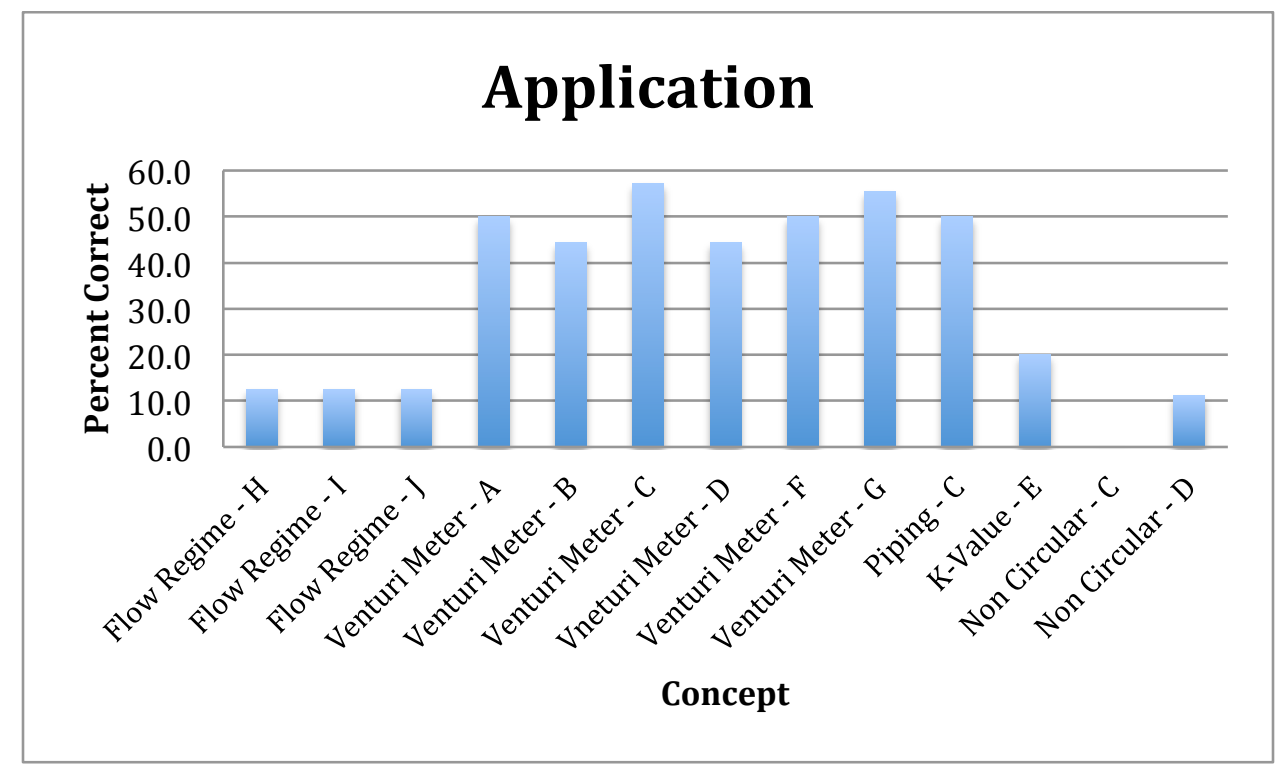

Figure 4: Percent correct answers of application questions asked in the interviews. Letters correspond to the interview questions under each category, identified in Table 2.

The quantitative results from student responses gives and initial gauge of retained student conceptual understanding in fluid mechanics and heat transfer. Further analysis of interview responses, however, can offer additional insights and information, revealing how students construct their understanding of a concept and how it fits into their existing conceptual understanding.

Results indicate the mechanical energy balance, the second major concept in the interview protocol, had poor results. Ten questions were used to address learning about the mechanical energy balance, with $6 \%$ of the responses for the first question on writing the mechanical energy balance correct. One student responded:

"Ok it definitely has the pressure drop on top, over the... pressure drop will have the pressure drop over the ... volumetric flow rate, volumetric flow rate, also have the friction loss, $h_{f}, u h h$, also have the energy... gosh I cant believe I forgot this equation, oh umm maybe also have the, um, shaft work in there. And energy loss from the system, pipe. Ok I don't remember something else." 
The student struggles through the response, even noting her own frustration at forgetting the equation. She identifies pieces of the ME balance but fails to offer a holistic understanding of all the energy contributions in the balance. Most of the responses determined to be incorrect for this first question were because of insufficient correct information, like the student quoted above. Follow-up questions probing comprehension and application of concepts surrounding the mechanical energy balance had more incorrect than correct responses, with similar reasons why responses were incorrect.

The Venturi meter analysis requires understanding of several concepts in fluid mechanics analysis. Half of the students correctly described the relationship between pressure and velocity in the Venturi meter as flow goes through the throat. During the course of the interview, one student corrected her incorrect initial response after applying the mechanical energy balance to the system, thinking through the problem:

"I don't know if that's a trick question. I mean I guess it's not a trick question, but... I'm wondering now if I was wrong before, because it seems to me that there shouldn't be any difference in energy between the two of them, so possible going back to my assumption that both increase from $A$ to $B$, one of those could be flipped, and that would be why there's no energy difference between point $A$ and B."

The strength observed in this response is the student notices her own mistake, indicating problem-solving skills even after expressing an initially incorrect understanding. Unfortunately, several students did not correct their initial incorrect response to how pressure and velocity behave through a Venturi meter when presented with the mechanical energy balance. Contradictions within their answers indicate incorrect holistic frameworks for the concepts; students did not articulate proper connections between pressure and flow work energy and between velocity and kinetic energy. These lost connections indicate an incorrect alignment with the conservation of energy.

The most difficult concepts based on student interview responses are associated with understanding the K-value energy loss coefficients and non-circular channels. Interview questions about these concepts primarily fall into Bloom's application and comprehension categories, which require higher-level thinking and analysis than the knowledge questions. With respect to K-values, all student responses fell below $40 \%$, with the lowest score, at $20 \%$ correct, from the application question regarding how to determine a K-value with a plot. The responses for non-circular channels were similar, with answers to comprehension questions below $40 \%$ and application questions below $15 \%$. For example, one student answered the last question on the interview protocol, asking about two different systems with the same hydraulic radius with the following response:

"So, in having the same equivalent diameters... I feel like this implies that... the ... either the, yeah I think, I'm just going to say that the velocity of the fluid in each pipe will be the same. I feel like the... from what I remember, the hydraulic radius... takes into account the extra... shear stress applied by the inner annulus ... and... and so based on that I would say that, so the annulus and outer pipe will be much larger than the typical circular tube, but with equivalent diameter it would take into account I feel like they're both going to have the same velocity." 
The student understands the hydraulic radius relates to shear stress, but is unable to articulate the implications of using different piping systems. His conclusion that both systems have the same velocity is justified with the geometry of the systems; this relates to the hydraulic radius and equivalent diameter but results in an incorrect conclusion about how the fluid would behave in the system.This analysis supports a piece-meal understanding of the hydraulic radius and its use. Asking him to extend his understanding into the equivalent diameter convolutes his already incomplete conceptual framework; without initial solid understanding of the concept, the student is unable to apply his current understanding or increase his understanding through analysis of non-circular channels.

The combined results from the interview responses and quantitative analysis offer insights into the most robust conceptual difficulties students still possess. First on this list are K-value friction loss coefficients, which had $26 \%$ correct, followed closely by noncircular channels with $21 \%$ correct and the mechanical energy balance with $33 \%$ correct. Results show poor responses for all questions in these categories, and they score lower than all other concepts. The concepts that fall into the middle of an understanding continuum include flow regimes with $51 \%$ correct and the Venturi meter and the piping systems, with 55\% of students who answered both sets of questions correctly. Students performed the best on questions regarding fittings, with the most correct responses coming from these questions and $94 \%$ of students answering correctly.

The analysis of the questions with Bloom's taxonomy indicates an inverse relationship between the difficulty of the question and number of correct answers. The knowledge questions that are the simplest on the hierarchy did yield several incorrect answers but overall were $63 \%$ correct. The comprehension questions that fall on the next higher step in Bloom's taxonomy ask questions more complex than knowledge but below application. In this category, students answered $43 \%$ of the questions correctly. Application questions, which require the deepest level of conceptual understanding, had student responses that were $32 \%$ correct. Results from these interviews can now inform assessment creation focused on the persisting conceptual difficulties from fluid mechanics.

Subsequent in-class activity worksheet design is intentionally including leading questions that drive at the application level of Bloom's hierarchy. For example, to progressively address concepts surrounding the understanding of the hydraulic radius, questions like the following are included in the assessment: (1) What causes the pressure drop in an annulus? (2) What causes pressure drop in general? (3) How does this relate to the hydraulic radius? The idea is for students to recognize that the source of energy loss, represented by pressure drop, is wall drag. In the two systems, the drag is different because of differing geometries, and the hydraulic radius reflects this physical phenomenon. In addition, since the in-class activities are interactive, the professor and TAs are available to help lead discussions and ask questions of the students. The bends and pipes cartridge is also equipped to help us address this concept because the channel is a square duct.

Similar methodology is being followed in design of the Venturi meter activity, with much emphasis placed on analyzing the mechanical energy balance at different points throughout the flow conduit with the changing diameter. The Venturi meter worksheet also includes a thorough 
analysis and connectivity between the observed behavior of pressure and velocity and how it relates to energy contributions within the mechanical energy balance. This will help communicate the effectiveness and importance of understanding the role of the balance in fluid mechanics analyses to students.

\section{Conclusions}

In our pedagogy, we are proposing use of hands-on active learning techniques to enhance student conceptual change in fluid mechanics and heat transfer. Two cartridges have been developed, an energy loss in bends and pipes, and a Venturi meter that each snaps into a base unit; subsequently, miniaturized systems of each are brought into the classroom. A previous implementation involved use of a demonstration-mode pedagogy, and the goal in this paper was to determine what conceptual difficulties persisted after students took a fluid mechancis and heat transfer course. Using this information, we designed a set of activities that accompany actual hands-on learning at the desktop that will address and assess reduction in those difficulties.

This study began with structured interviews of students after completing a fluid mechanics and heat transfer course that used the demonstration mode. The interview protocol was created to focus on questions that reveal conceptual understanding about seven topics typically taught in fluid mechanics. These were then analyzed qualitatively using Atlas ti and quantitatively using Bloom's hierarchy. Results from the interviews yield several insights about where students continue to have conceptual gaps in their understanding of fluid mechanics. Lowest-scoring concept questions were associated with K-value loss coefficients, noncircular channels, and the mechanical energy balance with $27 \%$ of student responses to the questions correct; these are followed by flow regimes, the Venturi meter and piping systems that have an average of 54\% correct. Scoring highest were questions regarding fittings where students answered $94 \%$ of the questions correctly. These findings informed the design of assessment materials to accompany the cartridges for a within-design study in the classroom.

The worksheets are completed and include intentionally leading questions to help students align the hands-on learning experience with their conceptual understanding. These will accompany the energy loss in bends and pipes and Venturi meter cartridges. Activities will be preceded by pretests on the concepts and followed by post-tests. A report on the results analyzed to date will be given in the ASEE presentation. Because this is a within design study where half of the students get lecture and the other half an activity for one topic and the groups are switched for the another topic, we will have a head-to-head comparison between lecture and DLM activity for the same groups of students taught in the two modes. We also plan for post DLM activity interviews that will be compared with those from the previous year to ascertain whether conceptual difficulties shown to persist from a previous implementation with a lecture/ demonstration mode of instruction are substantially reduced. Based on the findings of this study, future work includes further development of DLM cartridge activities and accompanying worksheets for communicating about other concepts like controlling resistance, continuity, parallel flow, balancing of drag, gravitational and buoyancy forces in particle beds, and calculation of pressure drops in complex systems.

\section{Acknowledgements}


We acknowledge support from the National Science Foundation through CCLI Grants and DUE1023121. We further acknowledge the staff and students of the Gene \& Linda Voiland School of Chemical and Bioengineering for support of the work to build, test, and more importantly implement the DLMs. We gratefully acknowledge the insights gained from our NSF collaborators in the WSU School of Civil \& Environmental Engineering, Andrew Easley (MS), and Professors Jennifer Adam and Shane Brown, who paved the way for the interview, assessment, and modified design implementation strategy. Finally, we acknowledge the important design contributions and manufacturing of the DLMs by Gary Held, Machinist in the WSU College of Engineering and Architecture Machine Shop.

\section{References}

${ }^{1}$ Felder, Richard, M. "Reaching the Second Tier - Learning and Teaching Styles in College Science Education,” J. Coll. Sci. Teaching, 23(5), 286-290, (1993).

${ }^{2}$ Kresta, Suzanne, M., "Hands-On Demonstrations: An alternative to Full Scale Lab Experiments," Jr. Engr. Education, 87(1), 7-9 (1998).

${ }^{3}$ Felder, R.M., Woods, D.R., Stice, J.E., Rugarcia, A., "The Future of Engineering Education: Teaching Methods that Work," Chem. Engr. Education, 34(1), 26-29, (2000).

${ }^{4}$ B.S. Bloom and D.R. Krathwohl, Taxonomy of Educational Objectives. Handbook 1: Cognitive Domain. Addison-Wesley, New York, 1984.

${ }^{5}$ Van Wie, B., et al., "Multi-disciplinary hands-on desktop learning modules and modern pedagogies," American Society for Engineering Education, 2012.

${ }^{6}$ Minerick, Adrienne, R., Schulz, Kirk H., "Freshman Chemical Engineering Experiment: Charged up on Electrophoresis." American Society for Engineering Education, 2012.

${ }^{7}$ Easley, Andrew; Adam, Jennifer; Brown, Shane; Montfort, Devlin; Van Wie, Bernard. "Open Channel Flow Misconceptions and Ontological Categories," Frontiers in Education Conference, Seattle, WA, Oct. 3-6, 2012.

${ }^{8}$ Easley, Andrew P.W., The Effectiveness of a Hands-on Desktop Module for Learning Open Channel Flow Concepts. December 2012. Thesis, Washington State University, Department of Civil and Environmental Engineering.

${ }^{9}$ Visco, Donald Jr., “Student Designed Desktop Modules in a Thermodynamics Course.” ASEE Annual Conference and Exposition, 2011.

${ }^{10}$ Miller, Streveler, \& Roman. Identifying and Repairing Student Misconceptions in Thermal and Transport Science: Concept Inventories and Schema Training Studies. Summer 2011.

Fundamental Research in Engineering Education, 45(3)., 


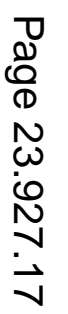

